\title{
Influenza-Like Symptom Prediction by Analyzing Self-Reported Health Status and Human Mobility Behaviors
}

\author{
Fenglong $\mathrm{Ma}^{*}$ \\ fenglong@buffalo.edu \\ Department of Computer Science and Engineering \\ University at Buffalo, The State University of New York \\ Buffalo, NY, USA \\ Jing Gao \\ jing@buffalo.edu \\ Department of Computer Science and Engineering \\ University at Buffalo, The State University of New York \\ Buffalo, NY, USA
}

\begin{abstract}
Human mobility behaviors are of great importance to predict influenza-like symptoms. However, most existing studies focus on analyzing population-level outcomes instead of individual-level. One challenge for individual-level influenza symptom prediction is a shortage of a sufficiently large dataset that contains individual health status as well as the mobility behavior information at the same time. Besides, the quality of the collected data is not high enough, due to the carelessness and low variation of reporting behaviors. Also, the number of individuals with influenza symptom onset is much smaller than that of ones without symptoms, i.e., the imbalanced data problem. These challenges further increase the difficulty of accurately predicting influenza-like symptoms. To address these challenges, in this paper, we propose a novel and powerful selective ensemble support vector machines (SESVM). The proposed SESVM can select the best basic SVM classifier by running on the randomly split sub training sets, which consist of the positive samples and the split negative ones. By randomly splitting the dataset multiple times, we can obtain many predictions by each best basic SVM classifier. SESVM finally aggregates all the predictions together to produce the final results. We conduct Experiments on a new longitudinal individual self-reported weekly survey dataset with mobility behaviors, and the results show that the proposed SESVM outperforms all the existing approaches for the influenza symptom prediction task.
\end{abstract}

\section{CCS CONCEPTS}

- Applied computing $\rightarrow$ Health informatics; • Information systems $\rightarrow$ Data mining.

\footnotetext{
${ }^{*}$ Both authors contributed equally to this research.

Permission to make digital or hard copies of all or part of this work for personal or classroom use is granted without fee provided that copies are not made or distributed for profit or commercial advantage and that copies bear this notice and the full citation on the first page. Copyrights for components of this work owned by others than ACM must be honored. Abstracting with credit is permitted. To copy otherwise, or republish, to post on servers or to redistribute to lists, requires prior specific permission and/or a fee. Request permissions from permissions@acm.org.

ACM-BCB '19, September 7-10, 2019, Niagara Falls, NY, USA

(c) 2019 Association for Computing Machinery.

ACM ISBN 978-1-4503-6666-3/19/09 . \$ $\$ 15.00$

https://doi.org/10.1145/3307339.3342141
}

\author{
Shiran Zhong* \\ shiranzh@buffalo.edu \\ Department of Geography \\ University at Buffalo, The State University of New York \\ Buffalo, NY, USA \\ Ling Bian \\ lbian@buffalo.edu \\ Department of Geography \\ University at Buffalo, The State University of New York \\ Buffalo, NY, USA
}

\section{KEYWORDS}

Healthcare informatics, human mobility behaviors, predictive models, ensemble models

ACM Reference Format:

Fenglong Ma, Shiran Zhong, Jing Gao, and Ling Bian. 2019. Influenza-Like Symptom Prediction by Analyzing Self-Reported Health Status and Human Mobility Behaviors. In 10th ACM International Conference on Bioinformatics, Computational Biology and Health Informatics (ACM-BCB '19), September 7-10, 2019, Niagara Falls, NY, USA. ACM, New York, NY, USA, 10 pages. https://doi.org/10.1145/3307339.3342141

\section{INTRODUCTION}

Mobility behaviors often put an individual in situations that might impact his/her health and well-being [24, 26, 28]. In particular, during an influenza epidemic outbreak, visits to certain places (e.g., workplaces, schools, or shopping malls) often expose an individual to a high risk of infection. Once infected, individuals might develop symptoms such as the runny nose, sour throat, headaches, and at times, other severe complications in 1-4 days [1]. Accurate prediction of individual symptoms based on their mobility behaviors is of paramount importance for the well-being, public health surveillance, and intervention strategy development, yet very few studies have investigated this issue. Given an accurate prediction, vulnerable individuals could be targeted and informed of early warnings as well as preventive tips. It also lays a solid foundation for surveillance of the epidemic outbreak at a population scale, so that policymakers could react to the potential outbreak promptly by allocating resources to the most vulnerable communities [3, 23].

In the past decade, an increasing amount of studies have started to investigate the association between human mobility and health. Most studies detect mobility patterns at a coarse scale and analyze the relevant impact on population-level health outcomes $[2,4,6$, $8,15]$. However, very few studies have focused on an individuallevel and explored the impact of mobility behaviors on the health status of individuals, since such studies usually encounter two key challenges. Firstly, existing work only uses a limited amount of mobility features of individuals (e.g., their total displacements, the radius of gyration, and the number of unique visited places) in health status prediction. Secondly, the reliability of results is mostly limited by the sample size due to an enormous amount 
of challenges in collecting individual-level data in a longitudinal manner [3, 9]. Thus, in order to accurately predict the influenza symptom onset of individuals based on their mobility behaviors, a more comprehensive and representative dataset that captures both the health information and mobility behavior of individuals simultaneously is much needed.

Besides, it is extremely hard for data collectors to totally control the quality of the data. In other words, the collected data contain a lot of noisy information. Although the collectors designed many questions which are highly related to the influenza-like symptom prediction task, some participants may submit their weekly reports carelessly and even wrong data. In addition, the behavior of participants prior to their symptom onset might be of little difference to their daily routine behavior, and such low variation in their behavior patterns and noisy information heavily increase the difficulty of the influenza-like symptom prediction task. Moreover, the number of individuals who have influenza-like symptoms (i.e., positive samples) is usually much smaller than that of ones without infecting influenza viruses (i.e., negative samples). This further results in the data imbalance problem. Thus, how to design an effective model to handle the low-quality and imbalanced data when predicting influenza-like symptoms is the key challenge.

To solve the aforementioned challenges, in this paper, we design a new and effective approach, called Selective Ensemble Support Vector Machines (SESVM), which not only perfectly handles the low-quality and imbalanced data but also significantly improves the prediction performance. Specifically, since the number of training positive data is limited, we first randomly split the training negative data into $M$ parts without any overlap. The positive data and each part of negative data become a new sub training set. The basic SVM runs on each sub training set and tests on the whole training set. We select the basic SVM classifier which performs the best on the training set, and then run it on the testing data to obtain a prediction vector. We can repeat the above procedure $T$ times to obtain $T$ prediction vectors on the testing data. Finally, we aggregate all the $T$ predictions with the majority voting to obtain the final results. The results on a real dataset show that the proposed SESVM outperforms all the baselines.

In summary, we have made the following contributions in this paper:

- We propose a novel and powerful model for tackling the challenges of influenza-like symptom prediction task, which selects the best basic classifier and further aggregates all the best predictions to guarantee the final performance.

- We take full advantage of a novel yet big dataset, which is collected via reporting weekly survey by participants' smartphones. The number of participants is more than 2,700 , and the collection lasts more than six months.

- We conduct extensive experiments on the real dataset to demonstrate the effectiveness of the proposed algorithm. Results show that the proposed algorithm achieves the best performance compared with existing algorithms.

The rest of the paper is organized as follows: Section 2 offers an overview of smartphone survey data, and Section 3 describes the data processing as there are a massive amount of features that might be potentially relevant to the prediction task. Section 4 illustrates the design of the proposed prediction model. Section 5 demonstrates the results from experiments. In Section 6, we briefly summarize the related studies. Section 7 concludes the paper.

\section{DATA \& PROBLEM}

The dataset is derived from a smartphone-based survey conducted during the 2016-2017 influenza season in the Western New York region. The survey intended to understand the association between the mobility behavior of individuals and the dispersion of influenza. In total, more than 2,700 participants were recruited for about 30 weeks (late October to late May). Three sets of information were collected throughout the survey period: demographic information, health status, and mobility information.

The demographic information of participants includes home address, workplace, gender, age group (18-35, 36-65, and over 65), household size, and employment status. The health status indicates whether the participants, along with their family members, co-workers, or face-to-face contacts have any influenza-like symptoms each week. It also recorded if participants took any protective actions, such as obtaining a flu shot, seeing a doctor or getting Over-The-Counter medicines. The mobility information consists of up to five frequently visited locations reported by each participant every week.

Since there are some participants that did not consecutively submit their weakly reports, in this paper, we only retain the data of participants who continuously reported more than 8 weeks. Besides, a few participants continuously reported influenza-like symptoms for more than 3 weeks. This is not reasonable because the symptoms of influenza usually last 1 to 2 weeks longer ${ }^{1}$. Thus, we remove such participants in the dataset. Finally, the total number of participants is 1,485 .

In the designed weekly survey, we ask the participants report up to five of their most frequently visited places during the last week. Since most participants submit up to three places, in this paper, we only use the top three reported places, instead of five places. In our dataset, the total number of locations reported by participants, including home, workplace and top 3 frequently visited places, is 11,349 in both Erie and Niagara County. As shown in Figure 1, each blue dot represents a location.

In order to capture the group-level human mobility behaviors, we use census block groups ${ }^{2}$ instead of individual locations. Each block group contains at least one location. The total number of census block groups is 829 in the dataset. Figure 2 shows the distribution of addresses within census block groups. The X-axis is the (log) number of locations in a census block group, and Y-axis represents the (log) number of census block groups. Though this is a longtail distribution, about $70 \%$ (584 out of 829 ) census block groups contain more than five reported addresses that are frequently visited by participants. Thus, we can use census block groups to capture human mobility behaviors.

Since influenza is not a longitudinal disease, and the recovering time for individuals after infecting with flu usually is short. Thus, we define our prediction task as follows:

\footnotetext{
${ }^{1}$ The 2009 H1N1 pandemic: summary highlights, Centers for Disease Control and Prevention, https://www.cdc.gov/h1n1flu/cdcresponse.htm.

${ }^{2}$ https://en.wikipedia.org/wiki/Census_block_group
} 


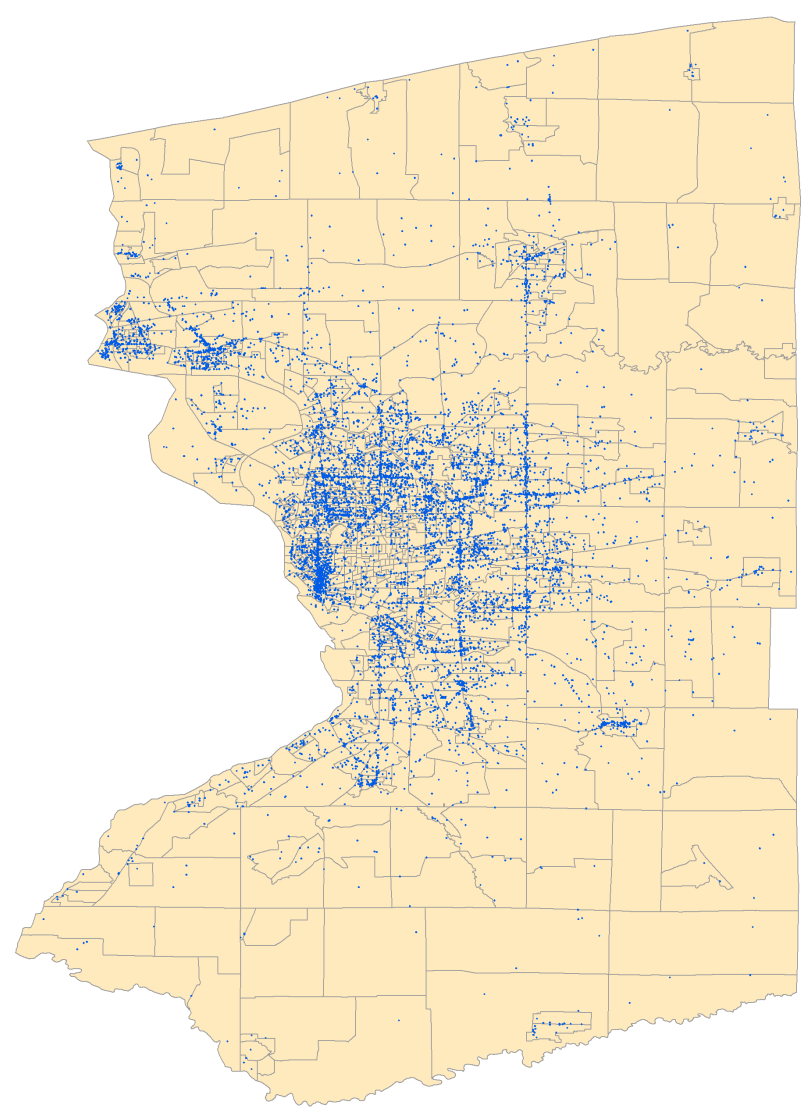

Figure 1: Reported Locations in Erie and Niagara County.

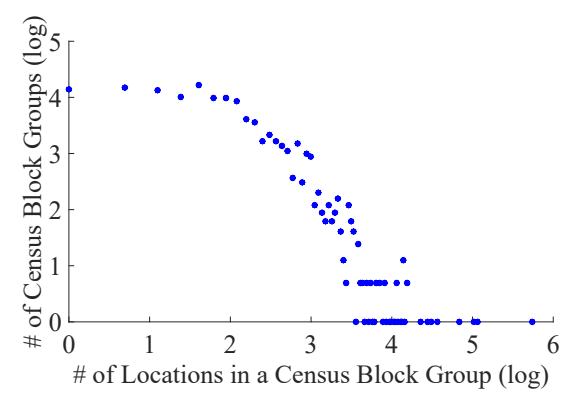

Figure 2: Log Distribution of Census Block Groups.

Problem. Given four weeks' self-reported survey data, our goal is to predict whether the participant will have influenza-like symptoms in the following one or two weeks as shown in Figure 3.

\section{FEATURE EXTRACTION}

The main goal of this paper is to study the relationship between human mobility behaviors of individuals and self-reported influenzalike symptoms. Towards this end, the first step is to extract a set of characteristics that systematically describe individuals, including both demographic information and mobility behaviors. Specifically, we divide the features into two categories: static features and dynamic features. In the following, the details about how to construct these features are introduced.

\subsection{Static Feature Extraction}

The static features refer to the characteristics of individuals that, loosely speaking, remain the same throughout the survey period. These features, such as age and residential communities, often put individuals at a different level of infection risk [14]. In this paper, we use such information as the set of static features. All the static features are summarized in Table 1 . Table 2 shows the statistics of different categorical static features.

\subsection{Dynamic Feature Extraction}

The dynamic features include two sets of information. The first set contains the characteristics associated with individuals that change over time, for example, the health status of an individual. The second set contains the activities that individuals took. These activities are assumed as the potential risk factors that lead to the change in individuals' health status. We extract dynamic features from multiple perspectives, including reviewing weekly reports provided by themselves, analyzing their contacting people, and even considering human mobility behaviors.

Self-Related Features. In the weekly survey data, there are some questions which are related to the health status of the participants themselves. In the following, we list the related items as features:

- I1: The participants were asked to indicate whether they displayed at least one of the following flu-like symptoms during the last week: Fever, runny nose, sore throat, cough, or nausea.

There are two ways to use this item. One is to obtain the ground truth data for the prediction task. The other is to consider the answer as a feature. Since the symptoms of influenza can last one or two weeks, if the participant had flu-like symptoms during the last week, then heshe may still have such symptoms in the current prediction period. The following five items (from I2 to I6), they are all protective actions for diseases, which may be helpful for the prediction:

- I2: Whether the participants had to go to work or school while sick during the last week.

- I3: Whether the participants stayed home sick from work or school during the last week.

- I4: Whether the participants went to the doctor during the last week.

- I5: Whether the participants took over-the-counter flu medicine during the last week.

- I6: Whether the participants have received a flu shot or mist as of today.

The proposed task is using the survey data of the previous four weeks to predict influenza-like symptoms of individuals in the following $m$ weeks. Thus, for the first five items (from I1 to I5), we use the accumulation of all the previous four weeks as the feature values. The reason is that we want to use these features to model the health status. If a person is very healthy and does not infect flu for a long time, then the probability of having influenza-like symptoms for this person may be low; otherwise, the probability is high. For the sixth item I6, we only use the current answer reported 


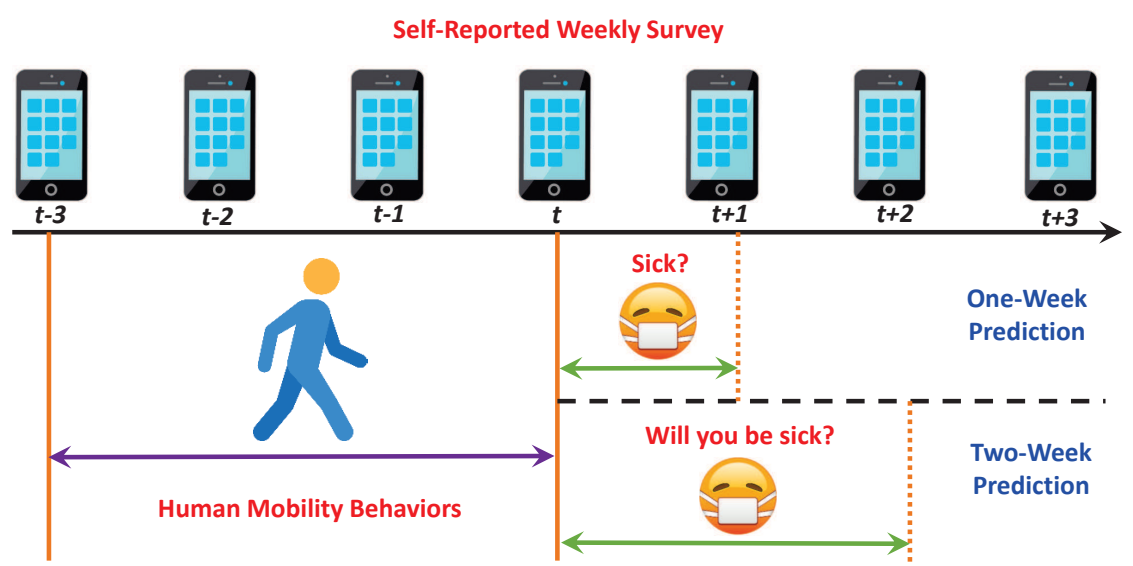

Figure 3: Task Overview. We use the survey data from week $t-3$ to week $t$ to extract features and predict whether the participant will have influenza-like symptoms in the week $t+1$ or $t+2$.

Table 1: Summary of Static Features.

\begin{tabular}{lll}
\hline Feature & Data Type & Description \\
\hline Gender & Categorical & Male or Female \\
Ethnicity & Categorical & Hispanic or Latino, not Hispanic or Latino \\
Race & Categorical & American Indian or Alaska Native, Asian, Black or African American, White, other race \\
Age Group & Categorical & $18-35,36-65,66$ or older \\
Minor 0-4 & Numerical & How many children in this household are from 0-4 years old \\
Minor 0-17 & Numerical & How many children in this household are from 0-17 years old \\
\hline
\end{tabular}

Table 2: Statistics of Categorical Static Features.

\begin{tabular}{c|l|c}
\hline Feature & Attribute & \# of Participants \\
\hline \multirow{2}{*}{ Gender } & Male & 486 \\
& Female & 999 \\
\hline \multirow{2}{*}{ Ethnicity } & Hispanic or Latino & 22 \\
& Not Hispanic or Latino & 1,463 \\
\hline \multirow{5}{*}{ Race } & American Indian or Alaska Native & 13 \\
& Asian & 34 \\
& Black or African American & 31 \\
& White & 1,390 \\
& Other Race & 17 \\
\hline \multirow{3}{*}{ Age Group } & $18-35$ & 542 \\
& $36-65$ & 905 \\
& 66 or older & 38 \\
\hline
\end{tabular}

in the last (fourth) week as the feature, and the value is 1 (Yes) or 0 (No).

Contacting Features. As a communicable disease, influenza is often transmitted through direct or indirect contact between the susceptible and the infectious. Specifically, when an infectious person coughs or sneezes, viruses are spread in a form of droplets or airborne. Infections occur when the viruses directly enter into the body of the nearby susceptible. Besides, a person might get flu by touching a surface or object that has flu viruses on it and then touching their own mouth, nose, or possibly their eyes. Thus, when predicting influenza-like symptoms, we should consider those important features.
The first category of frequently contacting people is family members. In the weekly survey, we can extract such features related to family members from the following items:

- I7: Whether any other adult living in the participants' household display flu-like symptoms during the last week.

- I8: Whether any child living in the participants' household display flu-like symptoms during the last week.

- I9: During the last week, whether the participants had to send their sick children to childcareschool.

- I10: During the last week, whether the participants kept their sick children at home from childcareschool.

- I11: During the last week, whether the participants took their sick children to the doctor.

- I12: During the last week, whether the participants gave their sick children over-the-counter flu medicine.

- I13: Whether the participants had all of the children living in their household received the flu shot or mist as of today.

The second category of possible contacting people is co-workers. We can extract a related feature from the survey:

- I14: Whether any co-worker of the participants displayed flu-like symptoms during the last week.

The third category is regarding face-to-face contacting persons. In the survey, we also consider such persons and extract a feature from the following item:

- I15: Whether anyone else the participants had face-to-face contact with displayed flu-like symptoms during the last week. 
For the aforementioned contacting features, from I7 to I15 (except I13), the values of those features are the accumulation values of the previous four weeks' survey data. According to [27], children younger than 18 are most likely to develop symptoms than adults. Therefore, we must consider the influence of children in the prediction model. Besides, if a person works in a crowded place, such as supermarkets or shopping malls, then he or she may contact with many people every day. Thus, they may have a high probability of contacting with influenza viruses. For the item I13, we use the fourth week's answer as the feature value, which is the same as I6.

Human Mobility Features. Human mobility behaviors are important factors for the prediction of influenza-like symptoms [3] When a person visits a place, the surrounding environment that he or she is exposed to has a great impact on his or her risk of infection, for example, how many people in the vicinity are recently infectious. In this study, the surrounding environment of a place is represented as the census block group, and the hazardousness of a block group is characterized by counting the number of symptomatic participants within the past two weeks. The temporal threshold of two weeks is set according to the possible infectious period of influenza [14]. In this view, the hazardousness of a block group is time-varying as the number of symptomatic participants in it changes with time. A participant is exposed to a different level of health risk as he or she travels to different census blocks in time. The total number of census block groups is 892 in the dataset, and we can obtain a vector with a length of 892 . If the participant visited a location within a census block group in the last two weeks (compared with $t$ ), then the corresponding value of that block group is 1 ; otherwise, it is 0 . Please note that the places a participant travels to include not only the top three frequently visited places, but also the home and workplace.

Time-Aware Features. Seasonality is of paramount importance in the influenza transmission. According to the CDC, seasonal influenza (flu) viruses in the United States are most common during the fall and winter. The exact timing and duration of flu seasons may vary a little, but most of the time, influenza activity begins to increase in October. Usually, flu activity peaks between December and February, although activity can last as late as May. Figure 4 from $\mathrm{CDC}^{3}$ shows peak flu activity in the United States by month for the 1982-1983 through 2017-2018 flu seasons. From Figure 4, we can observe that the time information is highly correlated to influenza.

In order to better predict the influenza symptom of participants throughout the survey period, we calibrate our model by integrating the seasonal background. We assume that the possibility of having symptoms, in general, is higher during the peak months while lower otherwise. Specifically, in this work, we divide the whole flu seasons into three levels. Level 1 includes October and November, Level 2 is from December to March, and the remaining months belong to Level 3. Thus, there are three time-aware features, which are extracted based on the fourth weekly report date. For example, the four dates are November 20, November 27, December 2 and December 7, then the time-aware feature for this participant in the current prediction window belongs to Level 2, i.e., the time-aware feature vector is $[0,1,0]$.

\footnotetext{
${ }^{3}$ https://www.cdc.gov/flu/about/season/flu-season.htm
}

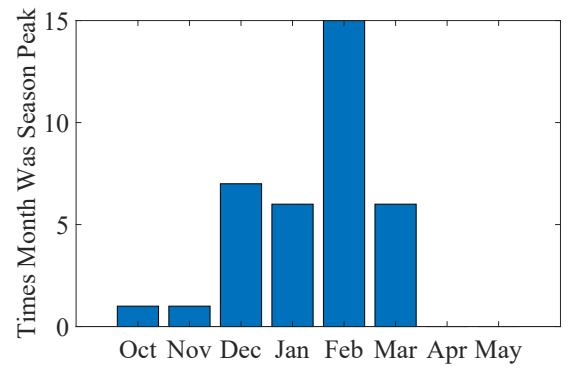

Figure 4: Peak Month of Flu Activity 1982-1983 through 2017-2018.

\section{METHODOLOGY}

Based on the extracted features in Section 3, We can design a classifier to predict whether a participant will have influenza-like symptoms according to the previous four weekly self-reported data. However, it is hard to achieve satisfactory performance for traditional classification algorithms, such as Logistic Regression [13], Random Forest [5] and Support Vector Machines (SVM) [12], which stems from the unique characteristics of the input data.

\subsection{Motivation \& Overview}

There are two types of limitations found in the dataset: data imbalance problem and low variation. Firstly, the self-related features refer to participants with symptoms (i.e., positive samples denoted by $\mathcal{N}^{+}$) and participants without symptoms (i.e., negative samples denoted by $\mathcal{N}^{-}$). Most of the time people are not infected and have no symptom, which leads to the data imbalanced problem, that is, the number of negative samples is much larger than that of positive ones. Secondly, the behavior of participants prior to their symptom onset might be of little difference to their daily routine behavior, and such low variation in their behavior pattern further adds difficulty in predicting their symptom onset.

These two limitations prevent us from applying traditional classification approaches on the dataset. For example, traditional ensemble model, Random Forest, can handle the problem of imbalanced data by constructing a multitude of decision trees at training time and outputting the class that is the mode of the classes of the individual trees. This approach considers all the results from all the individual classifiers. However, due to the low-quality of training data, the performance of most individual classifiers is unsatisfactory. When aggregating all results together, the overall performance drops. Though the overall quality of training data is low, some data still has the distinguishable ability, i.e., the performance of some individual classifiers is better than that of the overall performance. Thus, the challenge here is how to select distinguishable samples to train a good classifier.

\subsection{The Proposed SESVM}

To solve this challenge, we propose to use a novel result aggregation algorithm instead of directly selecting data. Specifically, we propose a new algorithm, Selective Ensemble Support Vector Machines(SESVM), which is built upon SVM with Radial Basis Function kernel (SVM-RBF). The proposed SESVM is an ensemble approach, 
but it is different from traditional ensemble models. SESVM first randomly divides negative data into $M$ parts. For each sub-dataset, we run SVM-RBF to train an individual classifier. We then evaluate the performance of the learned classifier on all the training data. Since there are $M$ individual classifiers, we store the prediction results outputted by the best individual classifier. It enables us to run the above process $T$ times. We can obtain $T$ best results. Finally, we use voting to get the final prediction. Next, the details of the proposed SESVM are introduced.

Data Partition. Let the number of positive training data be $\left|\mathcal{N}^{+}\right|$and the number of negative data in the training set be $\left|\mathcal{N}^{-}\right|$. It is worth noting that $\left|\mathcal{N}^{+}\right| \ll\left|\mathcal{N}^{-}\right|$. Since the dataset is extremely imbalanced, in order to train a good classifier, one possible way is to randomly select a subset of negative data to make the number of the negative samples be similar to that of positive data (i.e., $\left|\mathcal{N}^{+}\right|$). Assume that the whole negative samples $\mathcal{N}^{-}$can be partitioned into $M$ parts without overlapping, and $M=\left\lceil\frac{\left|\mathcal{N}^{-}\right|}{\left|\mathcal{N}^{+}\right|}\right\rceil$. Let $\mathcal{N}_{m}^{-}(1 \leq$ $m \leq M ; m \in \mathbb{N}_{+}$) denote the $m$-th partition of negative samples. Finally, we have $M$ sub training sets, i.e., $\left\{\mathcal{S}_{m}\right\}_{m=1}^{M}$, where $\mathcal{S}_{m}=$ $\left\{\mathcal{N}^{+} ; \mathcal{N}_{m}^{-}\right\}$.

Note that the proposed data partition procedure is different from the cross-validation technique. When we utilize cross-validation, the dataset is divided into multiple parts. Each partition contains both positive and negative samples. However, in this paper, only negative samples are divided, and we keep all the positive samples together.

Classifier Selection. In this paper, we use SVM-RBF as the basic classifier. On each sub-dataset $\mathcal{S}_{m}$, we can train a classifier $\Theta_{m}$. Hence, we can obtain $M$ individual classifiers. Different from traditional ensemble learning approaches directly applying $M$ classifiers on the testing dataset (denoted as $\mathcal{P}$ ), we apply them on the whole training dataset (i.e., $\mathcal{N}=\left\{\mathcal{N}^{+} ; \mathcal{N}^{-}\right\}$) to make classifier selection. For each basic classifier $\Theta_{m}$, we can make predictions on $\mathcal{N}$ and then calculate the prediction accuracy $a_{m} \in \mathbb{R}$. Finally, we can select the best classifier as follows:

$$
\Theta^{*}=\Theta_{k} ; k=\arg \max \left\{a_{1}, \cdots, a_{M}\right\}
$$

Result Aggregation. According to the selected classifier $\Theta^{*}$, we can make prediction the testing dataset $\mathcal{P}$. The prediction results are denoted as $\mathbf{P}^{*} \in\{0,1\}^{|\mathcal{P}|}$, where $|\mathcal{P}|$ is the number of samples in the testing set.

Due to the randomness of the data partition, the prediction vector $\mathrm{P}^{*}$ is also random. To reduce the influence introduced by randomness, we propose to run the data partition procedure $T$ times and then aggregate all the best predictions together. Let $\Theta_{t}^{*}$ represent the best selected classifier by the $t$-th iteration of the data partition, and $\mathbf{P}_{t}^{*}$ be the corresponding prediction vector. Based on the $T$ prediction vectors, i.e, $\mathbf{P}_{1}^{*}, \mathbf{P}_{2}^{*}, \cdots, \mathbf{P}_{T}^{*}$, we can use the majority voting (MV) strategy to obtain the final prediction denoted as:

$$
\hat{\mathbf{P}^{*}}=\operatorname{MV}\left(\mathbf{P}_{1}^{*}, \mathbf{P}_{2}^{*}, \cdots, \mathbf{P}_{T}^{*}\right)
$$

Algorithm 1 shows the whole procedure of the proposed SESVM.

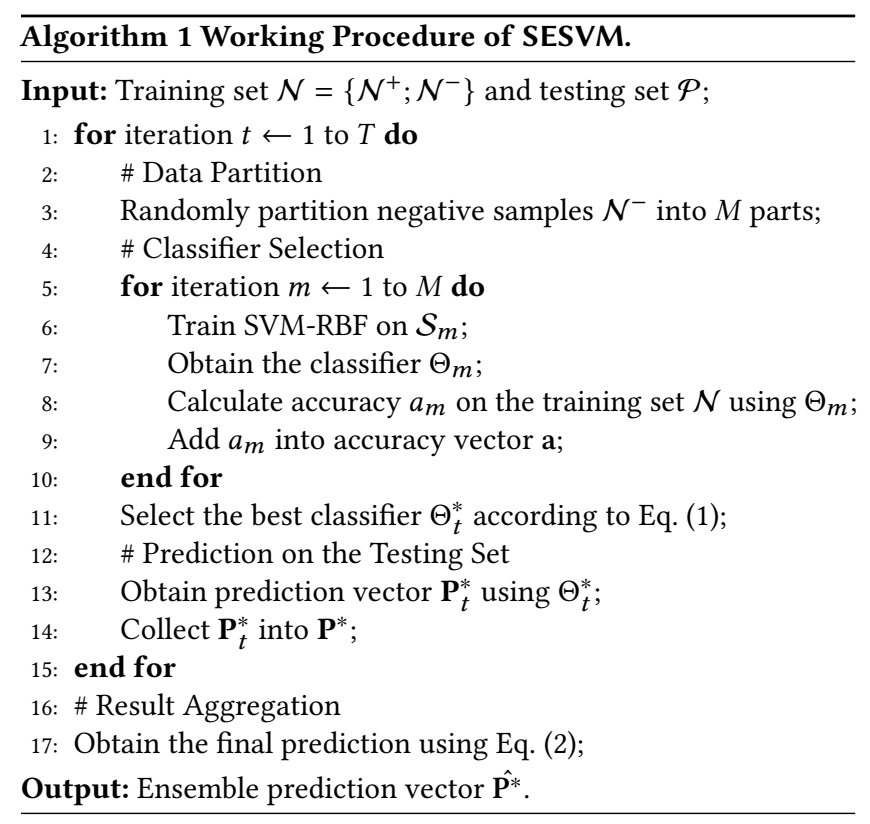

\section{EXPERIMENTS}

In this section, we first introduce the experimental setting, then show the performance of the proposed model compared with baselines, and finally conduct analysis to illustrate the motivation of the proposed approach.

\subsection{Experimental Setting}

The experimental setting includes the used datasets, baselines for performance comparison and evaluation measures.

Datasets. In our experiments, we aim to validate the prediction performance for influenza-like symptoms of individuals in the following one or two weeks given the previous four weeks' self-reported data. Thus, there are two datasets. One is used for one-week prediction (named POW dataset), and the other is to predict the next two weeks' health status (called PTW dataset). Table 3 lists the statistics of the two datasets.

Table 3: Statistics of the Two Datasets.

\begin{tabular}{cccc}
\hline Dataset & \# Samples & \# Positive & \# Negative \\
\hline POW & 25,221 & 2,756 & 22,465 \\
PTW & 23,736 & 4,316 & 19,420 \\
\hline
\end{tabular}

In order to validate the performance of the proposed model with a different amount of training data, we first randomly use $20 \%$ data as the testing set. The remaining data is used for training. In particular, we use $40 \%, 60 \%$, and $80 \%$ data as training sets, respectively. We incrementally add data into the training set, which means that $40 \%$ training dataset is a subset of both $60 \%$ and $80 \%$ training datasets, and $60 \%$ training set is the subset of $80 \%$ training one. Thus, the total number of datasets used is six in our experiments, and they all use the same testing dataset.

Baselines. We use Decision Tree, Logistic Regression, Random Forest and SVM as baselines. We implement all the algorithms with 
Table 4: Performance Evaluation on the Two Datasets with Different Training Ratios. "W" means that we use human mobility features, and " $\mathrm{W} / \mathrm{O}$ " indicates that we remove human mobility features from both datasets.

\begin{tabular}{c|c|cc|cc||cc|cc}
\hline \multirow{3}{*}{ Training Ratio } & \multirow{3}{*}{ Algorithm } & \multicolumn{3}{|c||}{ POW Dataset } & \multicolumn{4}{c}{ PTW Dataset } \\
\cline { 3 - 9 } & & \multicolumn{2}{|c|}{ Accuracy } & \multicolumn{2}{|c|}{ Weighted F1 } & \multicolumn{2}{c}{ Accuracy } & \multicolumn{2}{c}{ Weighted F1 } \\
\cline { 3 - 9 } 0 & & $\mathrm{~W}$ & $\mathrm{~W} / \mathrm{O}$ & $\mathrm{W}$ & $\mathrm{W} / \mathrm{O}$ & $\mathrm{W}$ & $\mathrm{W} / \mathrm{O}$ & $\mathrm{W}$ & $\mathrm{W} / \mathrm{O}$ \\
\hline \multirow{5}{*}{0.4} & Decision Tree & 0.6442 & 0.6458 & 0.7103 & 0.7117 & 0.6377 & 0.6384 & 0.6763 & 0.6769 \\
& Logistic Regression & 0.6406 & 0.6791 & 0.7068 & 0.7366 & 0.6005 & 0.6436 & 0.6449 & 0.6809 \\
& Random Forest & 0.7219 & 0.7391 & 0.7674 & 0.7778 & 0.6683 & 0.7030 & 0.7002 & 0.7248 \\
& SVM & 0.7403 & 0.6880 & 0.7787 & 0.7439 & 0.7091 & 0.6297 & 0.7273 & 0.6706 \\
& SESVM & $\mathbf{0 . 7 6 6 3}$ & 0.6896 & $\mathbf{0 . 7 9 3 2}$ & 0.7449 & $\mathbf{0 . 7 2 7 7}$ & 0.6430 & $\mathbf{0 . 7 3 5 3}$ & 0.6812 \\
\hline \multirow{5}{*}{0.6} & Decision Tree & 0.6458 & 0.6452 & 0.7116 & 0.7112 & 0.5390 & 0.5404 & 0.5885 & 0.5898 \\
& Logistic Regression & 0.6353 & 0.6791 & 0.7029 & 0.7366 & 0.6106 & 0.6443 & 0.6539 & 0.6816 \\
& Random Forest & 0.7068 & 0.7336 & 0.7571 & 0.7753 & 0.6759 & 0.6919 & 0.7069 & 0.7189 \\
& SVM & 0.7366 & 0.6704 & 0.7769 & 0.7308 & 0.7003 & 0.6371 & 0.7207 & 0.6766 \\
& SESVM & $\mathbf{0 . 7 6 3 9}$ & 0.6803 & $\mathbf{0 . 7 9 2 7}$ & 0.7382 & $\mathbf{0 . 7 1 6 5}$ & 0.6361 & $\mathbf{0 . 7 3 0 3}$ & 0.6753 \\
\hline \multirow{5}{*}{0.8} & Decision Tree & 0.6620 & 0.6624 & 0.7241 & 0.7244 & 0.6228 & 0.6224 & 0.6646 & 0.6642 \\
& Logistic Regression & 0.6357 & 0.6789 & 0.7034 & 0.7364 & 0.6051 & 0.6457 & 0.6493 & 0.6826 \\
& Random Forest & 0.7104 & 0.7138 & 0.7598 & 0.7615 & 0.6693 & 0.6883 & 0.7023 & 0.7175 \\
& SVM & 0.7292 & 0.6747 & 0.7714 & 0.7341 & 0.6959 & 0.6436 & 0.7182 & 0.6822 \\
& SESVM & $\mathbf{0 . 7 5 6 8}$ & 0.6783 & $\mathbf{0 . 7 8 7 9}$ & 0.7368 & $\mathbf{0 . 7 1 3 1}$ & 0.6441 & $\mathbf{0 . 7 2 8 3}$ & 0.6820 \\
\hline
\end{tabular}

scikit-learn $0.21 .0^{4}$. Since the dataset is imbalanced, all the algorithms are set the parameter class_weight as balanced. For Decision Tree, the maximum depth of the tree is 10. L-BFGS [7] is to use in the optimization of Logistic Regression. For Random Forest, the number of estimators is 100 , and the maximum depth of the tree is 10 . We use kernel SVM as the baseline, and the kernel is Radial basis function kernel. For the proposed SESVM, the parameters are the same as those of SVM. Besides, we set the number of iterations $T$ as 10 .

Evaluation Measures. To evaluate all the models, we use $A c$ curacy and Weighted F1 measures as the metrics. Since this is an imbalanced classification problem, the number of positive and negative samples is significantly different. Thus, we choose Weighted $F 1$ as the evaluation metric, which calculates metrics for each label and finds their average, weighted by support (the number of true instances for each label).

\subsection{Performance Evaluation}

Table 4 shows the performance of all the models on the two datasets with different training ratios. We can observe that the proposed SESVM achieves the best performance on different datasets when using the designed human mobility features.

Performance Analysis with Human Mobility Features. From Table 4, we can observe that among all the baselines, the performance of SVM is the best. Thus, in the proposed algorithm, SVM is the basic classifier. Due to the characteristic of imbalance, we first sample negative data and then train the basic classifier SVM on the whole training dataset. Finally, we use the best basic classifier to make predictions. We collect all the best prediction results of each time and further aggregate them together as the final prediction. In fact, the selected classifier is better than the original SVM which is trained on all the training data. Obviously,

\footnotetext{
${ }^{4}$ https://scikit-learn.org/stable/
}

the aggregation results are much better than those obtained by the original SVM. Thus, we can conclude that the ensemble method is better than a single classifier in our task.

The baseline Random Forest is an ensemble approach, and its basic estimator is Decision Tree. From Table 4, we can find that the performance of Random Forest is significantly better than that of Decision Tree. This again confirms the effectiveness of the ensemble approach when tackling the influenza-like symptom prediction task.

Though the proposed SESVM achieves the highest both accuracy and weighted F1, we also can observe that with the increase of training ratio, the performance slightly drops. This observation contradicts traditional findings, that is, adding training data will improve the performance. In traditional tasks, the quality of the training data is high. When we use more high-quality data to train a classifier, they help the classifier to be close to the accurate boundary. However, for our task, the characteristics of positive samples and negative samples are similar, which leads to the low-quality of training data. In such a case, increasing the number of training data also introduces a lot of noise. This noisy information can slightly reduce the performance.

Performance Analysis without Human Mobility Features. From Table 4, we can observe that the performance of the baseline Decision Tree slightly changes, which shows that when we train the classifier, the designed human mobility features do not hurt the performance. In contrast, for both Logistic Regression and Random Forest, when we remove those features, the performance improves. Especially for Random Forest, it can achieve comparable performance with SVM that is trained using human mobility features. For SVM, its performance dramatically drops when we remove human mobility features. Since the number of features reduces, the data is not so noisy that the performance of SESVM is slightly better than that of SVM. However, using the designed human mobility features and the proposed denoising approach, SESVM still performs the best among all the baselines with or without using those features. 


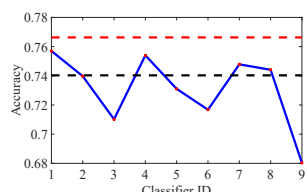

(a) Iteration 1

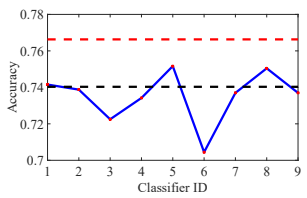

(f) Iteration 6

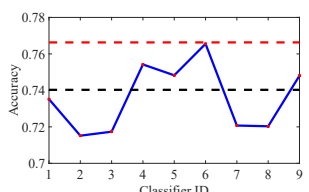

(b) Iteration 2

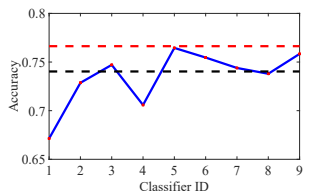

(g) Iteration 7

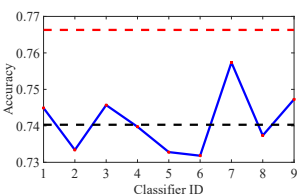

(c) Iteration 3

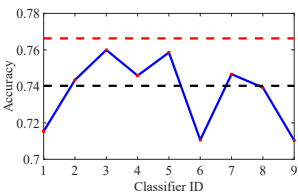

(h) Iteration 8

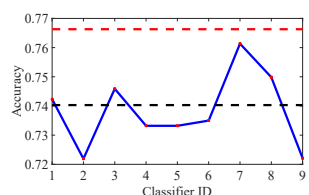

(d) Iteration 4

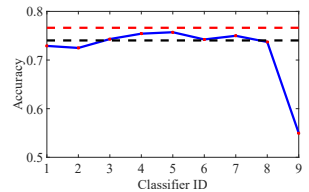

(i) Iteration 9

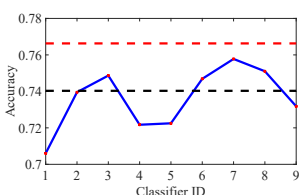

(e) Iteration 5

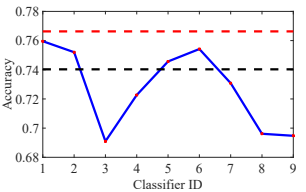

(j) Iteration 10

Figure 5: Illustration of Classifier Selection on the POW Dataset with Training Ratio $=0.4$.

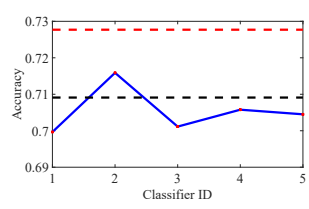

(a) Iteration 1

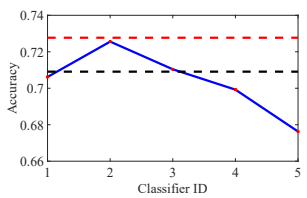

(f) Iteration 6

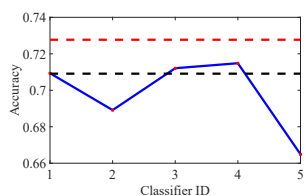

(b) Iteration 2

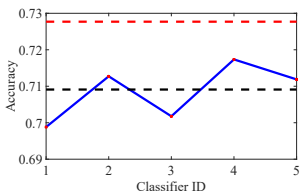

(g) Iteration 7

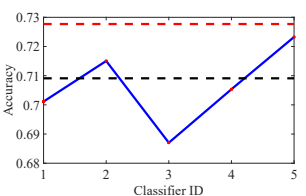

(c) Iteration 3

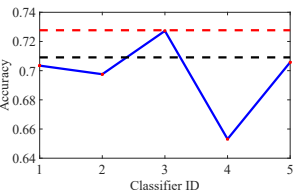

(h) Iteration 8

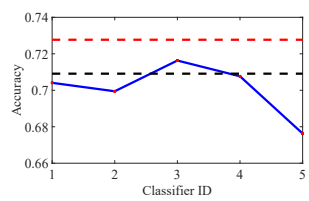

(d) Iteration 4

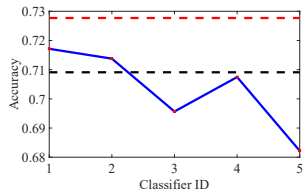

(i) Iteration 9

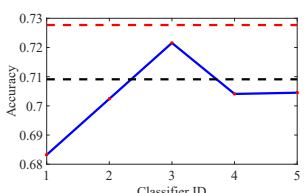

(e) Iteration 5

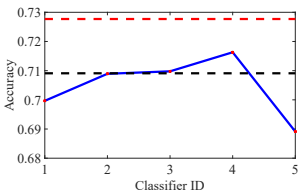

(j) Iteration 10

Figure 6: Illustration of Classifier Selection on the PTW Dataset with Training Ratio $=0.4$.

It clearly demonstrates the importance of considering mobility behaviors when predicting influenza-like symptoms with classifier SVM.

\subsection{Analysis of Classifier Selection}

In the proposed SESVM, there is an important module that aims to select the best classifier in the current iteration $t$. For the traditional ensemble approaches, they all directly put also the predictions made by basic classifiers together to produce the final prediction, such as Random Forest. Here, we conduct an experiment to show why this approach does not work for our task.

In this experiment, we first randomly partition the positives samples into $M$ parts. For the POW dataset, $M$ is 9 ; and $M=5$ for the PTW dataset. After obtaining $M$ new training sets, we can train $M$ classifiers and then make prediction $M$ times. To qualitatively demonstrate the benefit of designing the classifier selection module, we show $M$ accuracy obtained by those classifiers. We take the accuracy produced by both datasets with training ratio $=0.4$ as examples ${ }^{5}$, which are shown in Figure 5 and 6, respectively. The $\mathrm{X}$-axis is the ID of different basic classifiers, and Y-axis represents the corresponding accuracy.

${ }^{5}$ Note that we can obtain similar results on other datasets for both measures accuracy and weighted $\mathrm{F} 1$.
In Figure 5 and Figure 6, the dashed line in red color represents the accuracy of the proposed SESVM, the balck dashed line is the accuracy of SVM, and the blue line with red markers denotes the accuracy obtained by different basic SVM classifiers. To distinguish the baseline SVM and the individual basic estimator SVM used in SESVM, we use $\mathrm{bSVM}_{i}$ to represent the $i$-th basic classifier. We can observe that the performance of different basic classifiers (bSVMs) is different, which proves that the quality of training data varies largely. Compared with the baseline SVM, there are always a few bSVMs outperforming it in every iteration. However, we cannot guarantee that putting all the bSVM's predictions together can achieve better performance than SVM. Thus, the challenge is how to automatically choose representative samples to train a satisfactory classifier. To solve this challenge, we propose to select the best classifier in each iteration and then aggregate the results. Next, we demonstrate the effectiveness of result aggregation.

\subsection{Analysis of Result Aggregation}

Figure 7 shows the results of classifier selection (blue line) with the accuracy of SVM (black dashed line) and SESVM (red dashed line). The $\mathrm{X}$-axis denotes the number of iterations, and $\mathrm{Y}$-axis is the value of accuracy. We can observe that every accuracy from each basic classifier (i.e., bSVM) is higher than that of SVM, and the aggregation result of SESVM is better than that of each the basic 
classifier. From Figure 7, we can safely conclude that the proposed result aggregation is effective for the task influenza-like symptom prediction and significantly improves the prediction performance compared with the best baseline SVM.

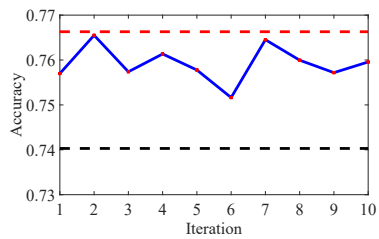

(a) POW Dataset

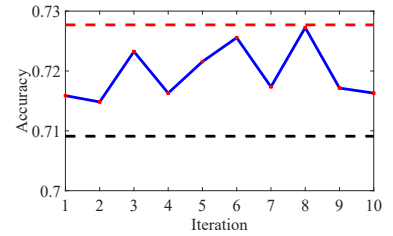

(b) PTW Dataset
Figure 7: Illustrating the Importance of Result Aggregation on Both Datasets with Training Ratio $=0.4$.

\subsection{Analysis of the Number of Iterations}

In the proposed SESVM, we can randomly partition the dataset $M$ times. We can calculate the aggregation results after each iteration. For example, when SESVM run five times, then we can use all the five outputs for result aggregation and calculate the accuracy and weighted F1. To show the performance changes in terms of iterations, we plot Figure 8.

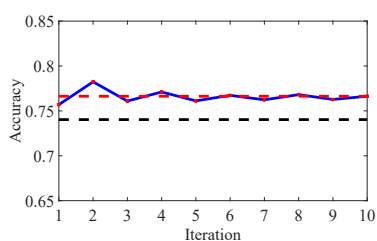

(a) POW Dataset

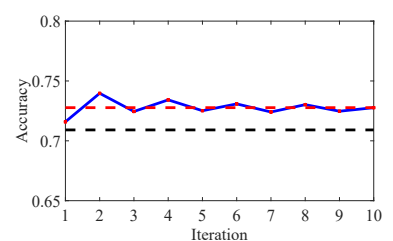

(b) PTW Dataset
Figure 8: Aggregation Accuracy Changes in Terms of Iterations on Both Datasets with Training Ratio $=0.4$.

In Figure 8, X-axis represents the number of iterations, and $\mathrm{Y}$ axis is the aggregation accuracy from all the $t$ iterations. The blue line denotes the aggregation accuracy of different iterations. The red dashed line is the accuracy of SVM, which is the aggregation results of all the ten iterations. The black dashed line is the accuracy of baseline SVM. From Figure 8, we can observe that the performance of the aggregation converges with the increase of the number of iterations, which demonstrates the robustness of the proposed SESVM.

\subsection{Discussions}

It is worth mentioning that thought there are many deep learning models for disease prediction [19-22, 25], in this paper, we do not use deep learning based models as baselines. The reason is that there is a lot of noisy information in the dataset. Even for deep learning models, they still cannot learn a good classifier. Besides, we extract two types of features: categorical and continuous features. For deep learning models, it is hard to combine different type's features together to train a model with good predictive ability. Although there are some studies working on how to combine features with different types, such as [11], due to the limited number and quality of data, it is hard to unleash the predictive power of deep learning models.

\section{RELATED WORK}

In this section, we briefly introduce studies that are related to influenza symptom prediction from two perspectives: predictions with human mobility data and imbalanced classification models.

\subsection{Prediction Models with Mobility Behaviors}

There are several studies to investigate the association between human mobility and human health [17]. Most of these studies focus on the mobility pattern at a coarse scale and study the impact on the population-level health. For example, studies have shown that the international airline transportation network significantly contributes to the increase of influenza incidence at world-wide large cities $[2,6,15]$. In addition, the routine daily commuting of population has also been identified highly correlated with the incidence rate at a county level $[4,8]$. Little research has explored the impact of individual mobility behaviors on their health status. The very few individual-level studies encounter two major challenges. Firstly, only a limited amount of mobility features of individuals are usually considered (e.g., the total displacements, the radius of gyration, and the number of unique visited places) in predicting the health status of individuals. Secondly, the reliability of results is largely limited by the sample size due to an enormous amount of challenges in collecting individual-level data in a longitudinal manner $[3,9]$. In order to accurately predict the influenza symptom onset of individuals based on mobility behaviors, a more comprehensive and representative dataset that captures the health information and mobility behavior of individuals is much needed. In this paper, we use a novel yet big dataset, which is collected via reporting weekly survey by participants' smartphones. The number of participants is more than 2,700 , and the collection lasts more than six months.

\subsection{Imbalanced Classification Models}

In most real-world prediction or classification, the data imbalance problem prevails in various disciplines such as disease diagnosis, image recognition, crime prediction or fraud detection [16]. It usually occurs when each class (true or false) does not make an equal portion in the dataset. Existing studies have suggested the necessities to adjust metrics or adopt sampling methods to guarantee the model performance [18]. Two common approaches are adopted to balancing the data in most studies: either by over-sampling instances of the minority class or under-sampling instances of the majority class [10]. Both approaches, if designed properly, should prevent classifiers from biased toward one class or the other. Limitation persists in practice. Oversampling might result in model overfitting, because it introduces duplications and limits the model flexibility in handling actual data with greater variance. Undersampling, in a similar mechanism, might end up in model underfitting, as important feature information, which should be included in the model training, might be left out. In this sense, an approach that can effectively balance the dataset while avoiding the overfitting and underfitting problems is imperative in this study to accurately predict the symptoms onset of individuals. Different from previous studies that directly applying existing classification models, in our work, we design a new approach SESVM, which not only selects the best basic classifier in each iteration, but also effectively aggregate predictions from basic classifiers to improve the final performance. 


\section{CONCLUSIONS}

Accurately predicting influenza-like symptoms of individuals is of great importance for the well-being, public health surveillance, and intervention strategy development. However, it is not very easy to predict influenza symptoms for individuals. First, collecting a large enough individual reported dataset including their mobility behaviors usually needs considerable effort. Second, due to the careless even wrong reporting, the quality of the collected data is low. Moreover, the data with a low variation of reporting behaviors increase the difficulty of the task. Third, predicting influenza symptoms is an imbalanced classification problem, since the number of individuals infecting by influenza viruses is much smaller than that of ones without influenza symptoms.

In this paper, to tackle these challenges, we propose an effective model, namely selective ensemble support vector machines (SESVM), which significantly improves the predictive performance. In particular, we first keep all the positive training data, but randomly split the negative training data into $M$ parts without any overlap to handle the imbalanced data issue. In this way, we can obtain $M$ sub training sets by putting positive training data and each split part of negative data. We train an SVM classifier on each sub training set and make predictions on the whole training data. We store the prediction vector on the testing set produced by the basic SVM that performs the best on the training set. By repeating the above procedure multiple iterations, we finally obtain the results by conducting majority voting on the stored prediction vectors of each iteration. In such a way, the prediction performance can be strongly guaranteed. We conduct extensive experiments on a real longitudinal individual self-reported weekly survey dataset to show the effectiveness of the proposed SESVM. Moreover, the experimental results demonstrate the importance of considering human mobility behaviors for influenza-like symptom prediction. Finally, qualitative and quantitative analyses are conducted to demonstrate the reasonableness and correctness of the proposed SESVM.

\section{ACKNOWLEDGMENTS}

Research reported in this publication was supported in part by the National Institute of General Medical Sciences of the National Institutes of Health under Award Number R01GM108731 and the US National Science Foundation under Grant Number IIS-1747614. The content is solely the responsibility of the authors and does not necessarily represent the official views of the National Institutes of Health and National Science Foundation. The use of the case data has been approved by the Institutional Review Board at the authors' institution.

\section{REFERENCES}

[1] [n.d.]. Key Facts About Influenza (Flu). https://www.cdc.gov/flu/about/keyfacts. htm. Accessed: 2019-05-22.

[2] Duygu Balcan, Vittoria Colizza, Bruno Gonçalves, Hao Hu, José J Ramasco, and Alessandro Vespignani. 2009. Multiscale mobility networks and the spatial spreading of infectious diseases. Proceedings of the National Academy of Sciences 106, 51 (2009), 21484-21489.

[3] Gianni Barlacchi, Christos Perentis, Abhinav Mehrotra, Mirco Musolesi, and Bruno Lepri. 2017. Are you getting sick? Predicting influenza-like symptoms using human mobility behaviors. EPf Data Science 6, 1 (2017), 27.

[4] Brooke A Bozick and Leslie A Real. 2015. The role of human transportation networks in mediating the genetic structure of seasonal influenza in the United States. PLoS pathogens 11, 6 (2015), e1004898.
[5] Leo Breiman. 2001. Random forests. Machine learning 45, 1 (2001), 5-32.

[6] John S Brownstein, Cecily J Wolfe, and Kenneth D Mandl. 2006. Empirical evidence for the effect of airline travel on inter-regional influenza spread in the United States. PLoS medicine 3, 10 (2006), e401.

[7] Richard H Byrd, Peihuang Lu, Jorge Nocedal, and Ciyou Zhu. 1995. A limited memory algorithm for bound constrained optimization. SIAM fournal on Scientific Computing 16, 5 (1995), 1190-1208.

[8] Segolene Charaudeau, Khashayar Pakdaman, and Pierre-Yves Boëlle. 2014. Commuter mobility and the spread of infectious diseases: application to influenza in France. PloS one 9, 1 (2014), e83002.

[9] Vivek Charu, Scott Zeger, Julia Gog, Ottar N Bjørnstad, Stephen Kissler, Lone Simonsen, Bryan T Grenfell, and Cécile Viboud. 2017. Human mobility and the spatial transmission of influenza in the United States. PLoS computational biology 13, 2 (2017), e1005382.

[10] Nitesh V Chawla, Nathalie Japkowicz, and Aleksander Kotcz. 2004. Special issue on learning from imbalanced data sets. ACM Sigkdd Explorations Newsletter 6, 1 (2004), 1-6.

[11] Heng-Tze Cheng, Levent Koc, Jeremiah Harmsen, Tal Shaked, Tushar Chandra, Hrishi Aradhye, Glen Anderson, Greg Corrado, Wei Chai, Mustafa Ispir, et al. 2016. Wide \& deep learning for recommender systems. In Proceedings of the 1st workshop on deep learning for recommender systems. ACM, 7-10.

[12] Corinna Cortes and Vladimir Vapnik. 1995. Support-vector networks. Machine learning 20, 3 (1995), 273-297.

[13] David R Cox. 1958. The regression analysis of binary sequences. Fournal of the Royal Statistical Society: Series B (Methodological) 20, 2 (1958), 215-232.

[14] Anthony E Fiore, Timothy M Uyeki, Karen Broder, Lyn Finelli, Gary L Euler, James A Singleton, John K Iskander, Pascale M Wortley, David K Shay, Joseph S Bresee, et al. 2010. Prevention and control of influenza with vaccines: recommendations of the Advisory Committee on Immunization Practices (ACIP), 2010. (2010).

[15] Rebecca F Grais, J Hugh Ellis, and Gregory E Glass. 2003. Assessing the impact of airline travel on the geographic spread of pandemic influenza. European journal of epidemiology 18, 11 (2003), 1065-1072.

[16] Nathalie Japkowicz and Shaju Stephen. 2002. The class imbalance problem: A systematic study. Intelligent data analysis 6, 5 (2002), 429-449.

[17] Justin Lessler and Derek AT Cummings. 2016. Mechanistic models of infectious disease and their impact on public health. American journal of epidemiology 183, 5 (2016), 415-422.

[18] Rushi Longadge and Snehalata Dongre. 2013. Class imbalance problem in data mining review. arXiv preprint arXiv:1305.1707 (2013).

[19] Fenglong Ma, Radha Chitta, Jing Zhou, Quanzeng You, Tong Sun, and Jing Gao. 2017. Dipole: Diagnosis prediction in healthcare via attention-based bidirectional recurrent neural networks. In Proceedings of the 23rd ACM SIGKDD international conference on knowledge discovery and data mining. ACM, 1903-1911.

[20] Fenglong Ma, Jing Gao, Qiuling Suo, Quanzeng You, Jing Zhou, and Aidong Zhang. 2018. Risk prediction on electronic health records with prior medical knowledge. In Proceedings of the 24th ACM SIGKDD International Conference on Knowledge Discovery \& Data Mining. ACM, 1910-1919.

[21] Fenglong Ma, Yaqing Wang, Houping Xiao, Ye Yuan, Radha Chitta, Jing Zhou, and Jing Gao. 2018. A General Framework for Diagnosis Prediction via Incorporating Medical Code Descriptions. In 2018 IEEE International Conference on Bioinformatics and Biomedicine (BIBM). IEEE, 1070-1075.

[22] Fenglong Ma, Quanzeng You, Houping Xiao, Radha Chitta, Jing Zhou, and Jing Gao. 2018. Kame: Knowledge-based attention model for diagnosis prediction in healthcare. In Proceedings of the 27th ACM International Conference on Information and Knowledge Management. ACM, 743-752.

[23] Marcel Salathe, Linus Bengtsson, Todd J Bodnar, Devon D Brewer, John S Brownstein, Caroline Buckee, Ellsworth M Campbell, Ciro Cattuto, Shashank Khandelwal, Patricia L Mabry, et al. 2012. Digital epidemiology. PLoS computational biology 8, 7 (2012), e1002616.

[24] Eleanor Setton, Julian D Marshall, Michael Brauer, Kathryn R Lundquist, Perry Hystad, Peter Keller, and Denise Cloutier-Fisher. 2011. The impact of daily mobility on exposure to traffic-related air pollution and health effect estimates. Journal of Exposure Science and Environmental Epidemiology 21, 1 (2011), 42.

[25] Qiuling Suo, Fenglong Ma, Giovanni Canino, Jing Gao, Aidong Zhang, Pierangelo Veltri, and Gnasso Agostino. 2017. A multi-task framework for monitoring health conditions via attention-based recurrent neural networks. In AMIA annual symposium proceedings, Vol. 2017. 1665.

[26] Michele Tizzoni, Paolo Bajardi, Adeline Decuyper, Guillaume Kon Kam King, Christian M Schneider, Vincent Blondel, Zbigniew Smoreda, Marta C González, and Vittoria Colizza. 2014. On the use of human mobility proxies for modeling epidemics. PLoS computational biology 10, 7 (2014), e1003716.

[27] Jerome I Tokars, Sonja J Olsen, and Carrie Reed. 2017. Seasonal incidence of symptomatic influenza in the United States. Clinical Infectious Diseases 66, 10 (2017), 1511-1518.

[28] Amy Wesolowski, Nathan Eagle, Andrew J Tatem, David L Smith, Abdisalan M Noor, Robert W Snow, and Caroline O Buckee. 2012. Quantifying the impact of human mobility on malaria. Science 338, 6104 (2012), 267-270. 